\title{
Cumplimiento de buenas prácticas de almacenamiento en hospitales nivel III especializada del Ministerio de Salud. Huancayo- 2018
}

Compliance with good practices of storage in level III specialized hospitals of Ministry of Health. Huancayo - 2018

Yomar Alvaro Perez Muñoz 1,a, Erika Lizbeth Vega Ataucusi 1,a, Diana Esmeralda Andamayo Flores 1,b

\section{RESUMEN}

Objetivo: Evaluar el nivel y porcentaje de cumplimiento de las Buenas Prácticas de Almacenamiento en hospitales nivel III especializada del Ministerio de Salud. Huancayo. Material y métodos: Estudio tipo básico, nivel descriptivo, prospectivo y transversal, realizado en el Hospital Regional Docente Materno Infantil El Carmen y el Hospital Docente Clínico Quirúrgico Daniel Alcides Carrión, empleando para la inspección el instrumento de registro autoinspeción de las Buenas prácticas de almacenamiento. Resultados: Los 2 hospitales cumplen un nivel medio (65\%) para todas las 9 áreas evaluadas como valor promedio en el almacenamiento, siendo el nivel alto entre $77 \%$ a $100 \%$ en el área de almacén, área de recepción, área de aprobados, área de baja y rechazados, área de devoluciones o rechazo y el área de despacho; también de nivel medio 50\% para el área de cuarentena y no cumplieron para las áreas de muestras, contramuestras y el área de embalaje. Conclusiones: Se cumplieron la mayoría de criterios de las Buenas prácticas de almacenamiento de productos farmacéuticos, dispositivos médicos y productos sanitarios, sin embargo, existen algunos criterios que deben mejorarse o cumplirse.

PALABRAS CLAVE: Buenas Prácticas de Almacenamiento, cumplimiento, hospitales.

\section{SUMMARY}

Objective: Assess the level and rate of compliance with good practices in level III specialized hospitals of Ministry of Health. Huancayo - 2018. Material and Methods:

Basic type study, descriptive level, prospective and cross-cutting, executed in El Carmen Maternal and Child Instructional Regional Hospital and The Surgical Clinical Instructional Regional Hospital Daniel Alcides Carrion, using for the inspection the instrument of registration self-inspection of Good storage practices. Results:

These two hospitals comply with an average level (65\%) to all the 9 areas evaluated as average value in storage, being the higher level between $77 \%$ to $100 \%$ in the storage area, reception a home area, approved area, low and rejected area, area of returns or rejection and a home office; besides the average level $50 \%$ for the quarantine area and did not fulfill for the samples sites, counters samples and bagging área. Conclusions:

The majority of the criteria with good practices of storage of pharmaceutical products was fulfilled, medical devices and health products, however there are some critirias that should be improved or achieved.

KEY WORDS: Good practices of storage, fulfillment, hospitals.

Facultad de Ciencias de la Salud, Universidad Privada De Huancayo Franklin Roosevelt. Huancayo, Perú.

Bachiller; ${ }^{\text {}}$ Docente, Doctora en Farmacia y Bioquimica. 


\section{INTRODUCCIÓN}

Los medicamentos son esenciales e indispensables en los servicios de atención sanitaria son componentes esenciales en muchos de los programas de prevención y tratamiento de enfermedades (1).

Uno de los principales problemas que afrontan las Droguerías - Distribuidoras en el Perú es la falta de condiciones apropiadas (temperatura y humedad controladas) para el correcto almacenamiento de los medicamentos siendo el Perú un país con un clima variado, por la misma geografía; los medicamentos muchas veces no logran soportar las variaciones de temperatura, humedad o presión $(2,3)$.

En nuestro País La Dirección General de Medicamentos Insumos y Drogas (DIGEMID), es la autoridad encargada del control sanitario de los productos farmacéuticos, verifica, garantiza el cumplimiento de la política Nacional de medicamentos, normas técnicas y administrativas, aprobadas por el Ministerio de Salud de las entidades públicas y privadas que realicen las actividades de registro, importación y exportación, producción, almacenamiento y distribución, publicidad e información de los productos farmacéuticos, para garantizar la calidad de los medicamentos, dispositivos médicos y productos sanitarios en el sistema de suministro cumpliendo con las Buenas Prácticas de Almacenamiento $(4,5)$.

Según la ley $N^{\circ} 29459$ - Ley de Productos Farmacéuticos, Dispositivos Médicos y Productos Sanitarios, en el Art. $22^{\circ}$ declara que para desarrollar sus actividades, las personas naturales o jurídicas, públicas o privadas que se dediquen a la importación, la distribución, el almacenamiento, la dispensación y el expendio de productos farmacéuticos, dispositivos médicos y productos sanitarios deben cumplir con los requisitos y condiciones sanitarias establecidas en el Reglamento respectivo y en las Buenas Prácticas de Almacenamiento, Buenas Prácticas de Dispensación y contar con la certificación correspondiente en los plazos que establece el Reglamento (6).

Las Buenas Prácticas de Almacenamiento (BPA) actualmente es muy importante dentro de la industria farmacéutica, ya que la calidad de un producto farmacéutico, dispositivos médicos y productos sanitarios desde su fabricación hasta su dispensación tiene que ser la misma; por ello los sistemas de almacenamiento contemplan instrucciones claras sobre la recepción y el ordenamiento de los productos, el mantenimiento de su Calidad (1).

Las Buenas Prácticas de Almacenamiento es parte del sistema de aseguramiento de acuerdo a los estándares de la calidad apropiada, siendo las BPA un conjunto de normas mínimas obligatorias que deben cumplir los establecimientos de importación, distribución, dispensación y expendio de productos farmacéuticos y afines respecto a las instalaciones, equipos y procedimientos operativos, destinados a garantizar el mantenimiento de las características y propiedades de los productos farmacéuticos siendo el Químico Farmacéutico hoy en día tenga que cumplir un rol importante en la implementación y mantenimiento de un sistema que garantice el cumplimiento de las BPA (7).

En América Latina países como Perú, Argentina, Venezuela, Chile y Bolivia han implementado normas para un adecuado almacenamiento y distribución de los medicamentos para garantizar al paciente un producto de calidad (8).

La verificación del cumplimiento de las Buenas Prácticas de Almacenamiento (BPA) y demás normas vigentes se realizan a través de las inspecciones, desarrolladas sobre la base de Actas de inspección aprobadas por el Ministerio de Salud; con el fin de comprobar la observación de las normas de (BPA) dando garantía y calidad de que los productos de forma segura para reducir al máximo los riesgos de afectar la calidad de los productos farmacéuticos, dispositivos médicos y productos sanitarios.

El objetivo del presente trabajo fue eEvaluar el nivel y porcentaje de cumplimiento de las Buenas Prácticas de Almacenamiento en hospitales nivel III especializada del MINSA- Huancayo.

\section{MATERIAL Y METODOS}

Esta investigación corresponde a un estudio de tipo básico, que se caracterizará por fundamentar la práctica de las Buenas Prácticas de Almacenamiento en los hospitales nivel III, especializado.

La investigación fue de tipo descriptivo cuantitativo, diseño descriptivo de corte transversal con mediciones de los procesos operativos de aseguramiento de la calidad (PAC) así como de procedimientos operativos de almacén (9). 
La presente investigación fue de nivel descriptivo-cuantitativo porque precisará información sobre el cumplimiento de las Buenas prácticas de Almacenamiento.

El diseño de la investigación fue descriptivo de corte transversal con 3 mediciones de los procesos operativos de las Buenas Practicas de Almacenamiento en los hospitales nivel III especializados de Huancayo.

\section{Población de Estudio}

Para desarrollar esta investigación se cumple con definir la unidad de análisis a utilizar y la población que a continuación se detalla:

\section{Unidad de Análisis}

Fueron el cumplimiento de las buenas prácticas de almacenamiento en el Hospital Regional Docente Materno Infantil El Carmen y el Hospital Docente Clínico Quirúrgico Daniel Alcides Carrión, donde se inspeccionaron 9 áreas (tabla 1).

\section{Población de estudio}

Hospitales de nivel III especializados del MINSA del distrito de Huancayo a quienes se evaluó el cumplimiento de los procesos de Buenas Prácticas de Almacenamiento.

La muestra de investigación fue probabilística o intencionada en el Hospital Regional Docente Materno Infantil El Carmen y el Hospital Docente Clínico Quirúrgico Daniel Alcides Carrión.

\section{Técnica e Instrumentos de Recolección de Datos}

La técnica utilizada fue la observación estructurada, con la finalidad de cumplir mediciones de verificación sobre el cumplimiento de los procesos de las Buenas Practicas de Almacenamiento cada quince días y utilizando como instrumento el registro de auto inspección que consta de lo siguiente:

1. Áreas: Delimitadas, ordenadas, rotuladas

2. Infraestructura

3. Organización Interna

4. Recursos Humanos

5. Recursos Materiales

6. Seguridad y mantenimiento

7. Técnicas de manejo y distribución

Luego de recolectarse los datos de la investigación, por medio de la aplicación del instrumento, se procedió a construir una base de datos, haciéndose uso del programa Excel Versión 2013.

\section{RESULTADOS}

Con respecto a la inspección de los almacenes de los hospitales del MINSA, se puede apreciar en el gráfico 1 , que cumplen en nivel medio (65\%) para todas las 9 áreas evaluadas.

Sin embargo, se encontró un nivel alto con niveles de cumplimiento entre 77 a 100\% para las áreas de almacén, área de recepción, área de aprobados, área de baja y rechazados, área de devoluciones o rechazo y el área de despacho.

Tabla 1. Areas inspeccionadas.

\begin{tabular}{ll}
\hline Número & AREAS \\
\hline I & ÁREA ÁLMACEN \\
II & ÁREA DE RECEPCIÓN \\
II & ÁREA DE CUARENTENA \\
IV & ÁREA DE MUESTRAS Y CONTRAMUESTRAS \\
V & ÁREA DE APROBADOS \\
VI & ÁREA DE BAJA Y RECHAZADOS \\
VII & ÁREA DE DEVOLUCIONES O RECHAZO \\
VIII & ÁREA DE EMBALAJE \\
IX & ÁREA DE DESPACHO \\
\hline
\end{tabular}


También se encontró un nivel medio 50\% para el área de cuarentena.

Se hallaron un nivel de cumplimiento de bajo en $0 \%$ para las áreas de muestras y contramuestras y el área de embalaje.

En la gráfico 2, se puede apreciar que, al aplicar la medición de los indicadores de almacén, se encuentra que se cumplieron 12 criterios como la existencia de la recepción, cuarentena, muestras de retención, aprobados/almacenamiento, bajas/ rechazados, devoluciones, embalaje, despacho, productos controlados, área administrativa, servicios higiénicos, vestidores, material de limpieza y no cumpliéndose 1 criterio como la muestra de retención. En consecuencia existe un nivel de cumplimiento del $77 \%$.

En la tabla 2, de acuerdo a la medición del cumplimiento de las buenas prácticas de almacenamiento en el área de recepción se tiene que cumplieron el 100\% ( $\mathrm{n}=5)$ de los criterios ya sea: Elárea de recepción está separada, delimitada, identificada y

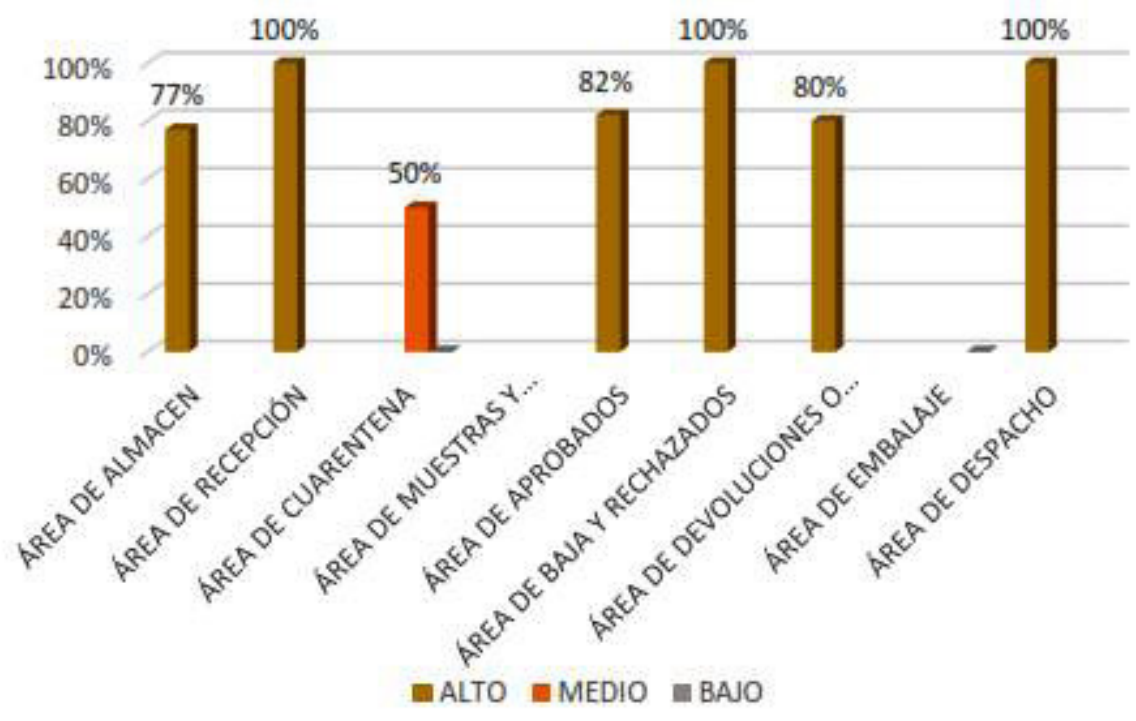

Grafico 1. Nivel y cumplimiento de las buenas prácticas de almacenamiento en las diferentes áreas de los hospitales especiales del MINSA.

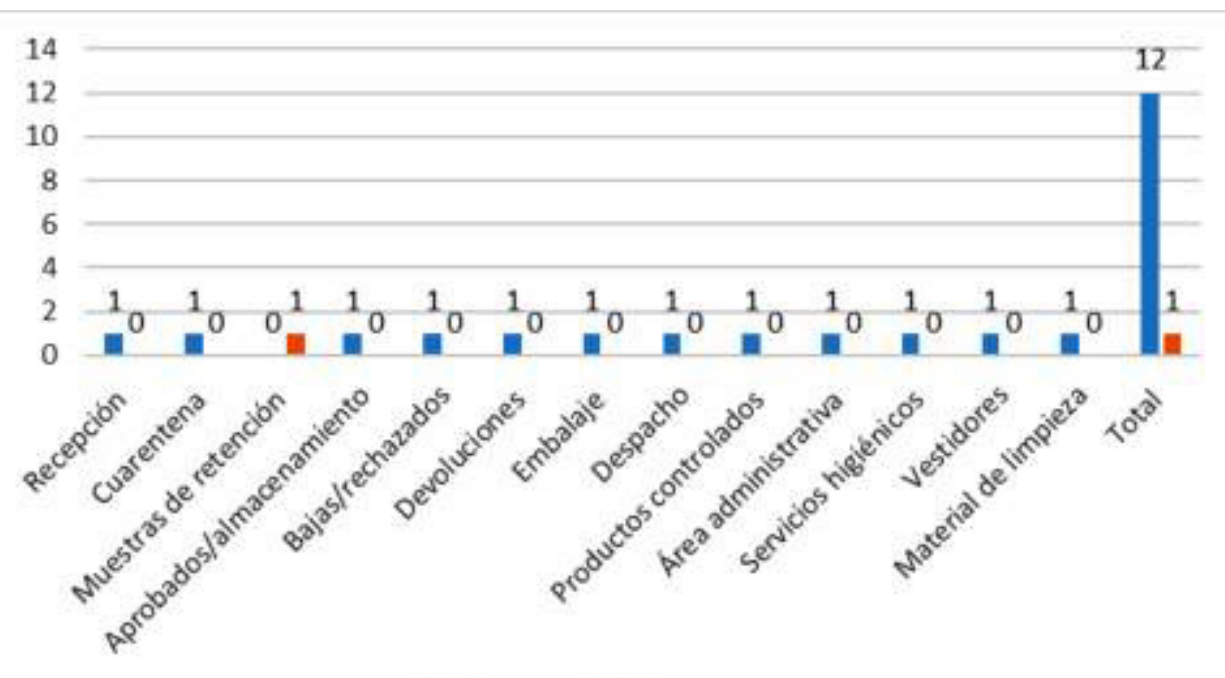

- CUMPLE NOCUMPLE

Gráfico 2. Medición del cumplimiento de las buenas prácticas de almacenamiento en el Area de Almacén. 
equipada; cuenta con los procedimientos escritos para la recepción, cada producto ingresa con su respectiva documentación, se realiza la revisión del estado del embalaje, en la recepción, se verifica como mínimo, nombre, concentración, fecha de vencimiento y forma farmacéutica del producto farmacéutico. Por tanto, el porcentaje de cumplimiento para las Buenas Prácticas de Almacenamiento en el área de recepción fue del $100 \%$.

En la tabla 3, en lo que respecta al cumplimiento de las buenas prácticas de almacenamiento en el área de cuarentena se tiene que de 4 criterios, solo se cumplieron 2 como: Cuenta con un área separada, delimitada e identificada y si se realiza la verificación del registro de recepción certificado de análisis. Obteniéndose un porcentaje de cumplimiento de Buenas Prácticas de Almacenamiento en el área de cuarentena del $50 \%$.
En la tabla 4, se puede apreciar de acuerdo a la medición del área para muestras y contramuestras se encuentra que no cumplen el único indicador: Cuenta con un área separada, delimitada, identificada y registrada.

Se tiene un porcentaje de cumplimiento de buenas prácticas de almacenamiento en el área de muestras y contramuestras del $0 \%$.

En el gráfico 3, se puede observar que en el área de aprobados del almacén de hospitales del MINSA se cumplieron 9 criterios de un total de 11. Habiéndose cumplido los criterios de: Cuenta con un área separada, delimitada e identificada, si los productos que requieren controles especiales se almacenan con las debidas medidas de seguridad, está documentado la altura de la estiva, la disposición de los productos farmaceuticos, dispositivos médicos

Tabla 2. Medición del cumplimiento de las buenas prácticas de almacenamiento en el área de recepción.

\begin{tabular}{lccc}
\hline Area de Recepción & Cumple & No cumple & $\begin{array}{c}\text { Porcentaje de } \\
\text { Cumplimiento }\end{array}$ \\
\hline $\begin{array}{l}\text { El Area de recepción está separada, } \\
\text { delimitada, identificada y equipada }\end{array}$ & 1 & 0 & \\
$\begin{array}{l}\text { Cuenta con los procedimientos escritos para la } \\
\text { recepción }\end{array}$ & 1 & 0 & \\
$\begin{array}{l}\text { Cada producto ingresa con su respectiva } \\
\text { documentación }\end{array}$ & 1 & 0 & $100 \%$ \\
$\begin{array}{l}\text { Se realiza la revisión del estado del embalaje } \\
\begin{array}{l}\text { En la recepción, se verifica como mínimo el } \\
\text { nombre, concentración, fecha de vencimiento }\end{array}\end{array}$ & 1 & 0 & \\
y forma farmaceutica del producto & 1 & 0 & \\
framaceútico & & & \\
TOTAL & 5 & 0 & \\
\hline
\end{tabular}

Tabla 3. Medición del cumplimiento de las buenas prácticas de almacenamiento en el área de cuarentena.

\begin{tabular}{lccc}
\hline Area de Cuarentena & Cumple & No cumple & $\begin{array}{c}\text { Porcentaje de } \\
\text { Cumplimiento }\end{array}$ \\
\hline $\begin{array}{l}\text { Cuenta con un área separada, delimitada, } \\
\text { identificada y equipada }\end{array}$ & 1 & 0 & \\
$\begin{array}{l}\text { Se realiza la verificación del registro de } \\
\text { recepción certificado de análisis }\end{array}$ & 1 & 0 & $50 \%$ \\
$\begin{array}{l}\text { En caso de productos termosensibles se } \\
\text { verifica el registro de temperatura }\end{array}$ & 0 & 1 & \\
$\begin{array}{l}\text { Se realiza la evaluación organoléptica de los } \\
\text { productos }\end{array}$ & 0 & 1 & \\
TOTAL & 2 & 2 & \\
\hline
\end{tabular}


Tabla 4. Medición del cumplimiento de las buenas prácticas de almacenamiento en el área de cuarentena.

\begin{tabular}{lccc}
\hline Area de cuarentena. & Cumple & No cumple & $\begin{array}{c}\text { Porcentaje de } \\
\text { Cumplimiento }\end{array}$ \\
\hline $\begin{array}{l}\text { Cuenta con un área separada, delimitada, } \\
\text { identificada y restringida }\end{array}$ & 0 & 1 & $0 \%$ \\
TOTAL & 0 & 1 & \\
\hline
\end{tabular}

Gráfico 3. Medición del cumplimiento de las buenas prácticas de almacenamiento en el Area de Aprobados.

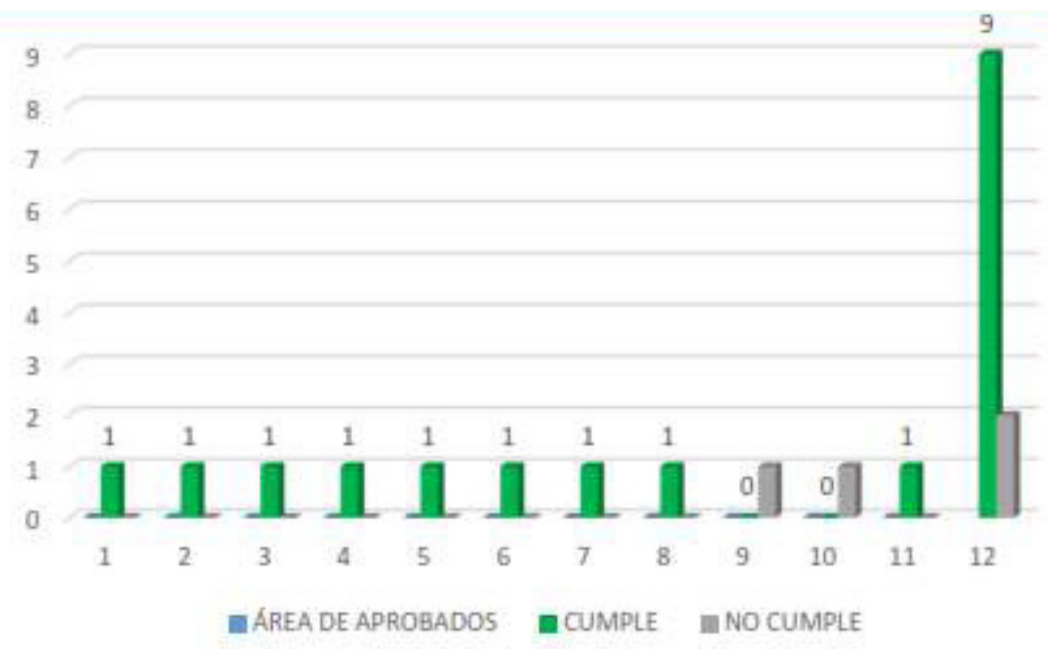

y productos sanitarios está hecho en base orden alfabético, forma farmacéutica, clase terapéutica, código del producto, tiene un registro de existencias que consignan el lote, código, fecha de vencimiento según corresponda de cada producto, cuenta con procedimientos operativos escritos sobre el control de existencias mediante inventarios, existe un sistema de alerta de los productos con fecha de vencimiento, se registra la investigación en caso se establezcan diferencias en el inventario.

Los productos almacenados se encuentran asegurados para evitar su caída, si cuentan con procedimientos operativos escritos para el almacenamiento incluyendo las condiciones del almacenamiento.

Por tanto existe un porcentaje de cumplimiento de Buenas Prácticas de Almacenamiento en el 82\%.

En la tabla 5, se puede apreciar en cuanto a las buenas prácticas de almacenamiento del área de baja o rechazados se tiene que cumplen los 3 indicadores establecidos como: Cuenta con un área separada, delimitada, identificada y restringida, cuenta con procedimientos operativos escritos por el proceso de baja de productos incluyendo la destrucción y se comunica a la autoridad sanitaria de su jurisdicción la destrucción de productos.

Por tanto, existe un porcentaje de cumplimiento de buenas prácticas de almacenamiento en el área de baja o rechazados del $100 \%$.

En la tabla 6 se puede apreciar que, en el área de devoluciones o rechazo, se cumplieron 4 indicadores de los 5 existentes como: Cuenta con un área separada, delimitada, identificada y restringida, cuenta con procedimientos operativos para el manejo de devoluciones, se registran, evalúan y documentan las devoluciones y sus causas, se registran los resultados y las medidas adoptadas y se asegura que los productos termo sensibles procedentes de devoluciones mantengan la cadena de frio.

Se obtiene un porcentaje de cumplimiento en el área de devoluciones del $80 \%$.

Enlatabla 7 sepuedeapreciar, encuantoal cumplimiento de las buenas prácticas de almacenamiento en el área de embalaje se puede apreciar que los hospitales del 
Tabla 5. Medición del cumplimiento de las buenas prácticas de almacenamiento en el área de baja y rechazados.

\begin{tabular}{lccc}
\hline Area de baja y rechazados & Cumple & No cumple & $\begin{array}{l}\text { Porcentaje de } \\
\text { Cumplimiento }\end{array}$ \\
\hline $\begin{array}{l}\text { Cuenta con un área separada, delimitada, } \\
\text { identificada y restringida }\end{array}$ & 1 & 0 & \\
$\begin{array}{l}\text { Cuenta con los procedimientos operativos escritos } \\
\text { por el proceso de baja de productos incluyendo la } \\
\text { destrucción }\end{array}$ & 1 & 0 & $100 \%$ \\
$\begin{array}{l}\text { Se comunica a la autoridad sanitaria de su } \\
\text { jurisdicción la destrucción de productos }\end{array}$ & 1 & 0 & \\
TOTAL & 3 & 0 & \\
\hline
\end{tabular}

Tabla 6. Medición del cumplimiento de las buenas prácticas de almacenamiento en el área de devoluciones o rechazo.

\begin{tabular}{|c|c|c|c|}
\hline Area de devoluciones o rechazo & Cumple & No cumple & $\begin{array}{l}\text { Porcentaje de } \\
\text { Cumplimiento }\end{array}$ \\
\hline $\begin{array}{l}\text { Cuenta con un área separada, delimitada, } \\
\text { identificada y restringida }\end{array}$ & 1 & 0 & \multirow{6}{*}{$100 \%$} \\
\hline $\begin{array}{l}\text { Cuenta con los procedimientos operativos para el } \\
\text { manejo de devoluciones }\end{array}$ & 1 & 0 & \\
\hline $\begin{array}{l}\text { Se registran, evaluan y documentan las } \\
\text { devoluciones y causas }\end{array}$ & 1 & 0 & \\
\hline Se registran los resultados y las medidas adoptadas & 1 & 0 & \\
\hline $\begin{array}{l}\text { Se asegura que los productos termo sensibles } \\
\text { procedentes de devoluciones mantengan la cadena } \\
\text { de frio }\end{array}$ & 0 & 1 & \\
\hline TOTAL & 4 & 1 & \\
\hline
\end{tabular}

Tabla 7. Medición del cumplimiento de las buenas prácticas de almacenamiento en el área de Embalaje.

\begin{tabular}{|c|c|c|c|}
\hline Area de Embalaje & Cumple & No cumple & $\begin{array}{l}\text { Porcentaje de } \\
\text { Cumplimiento }\end{array}$ \\
\hline $\begin{array}{l}\text { Cuenta con un área separada, delimitada, } \\
\text { identificada y restringida }\end{array}$ & 0 & 1 & \multirow{6}{*}{$0 \%$} \\
\hline $\begin{array}{l}\text { Cuenta con los procedimientos operativos para } \\
\text { embalaje }\end{array}$ & 0 & 1 & \\
\hline Se evaluan los factores de embalaje & 0 & 1 & \\
\hline Condiciones de conservacion del producto & 0 & 1 & \\
\hline $\begin{array}{l}\text { Se embala los productos de acuerdo al } \\
\text { procedimiento escrito }\end{array}$ & 0 & 1 & \\
\hline $\begin{array}{l}\text { El embalaje cuenta con rótulo indicando el manejo } \\
\text { de identificación para transporte e identificación }\end{array}$ & 0 & 1 & \\
\hline
\end{tabular}

MINSA, no cumplieron ningún criterio como: Cuenta con un área separada, delimitada, e identificada, cuenta con procedimientos operativos para embalaje, se evalúa los factores de embalaje, existe condiciones de conservación del producto, se embala los productos de acuerdo al procedimiento escrito y el embalaje cuenta con rotulo indicado el manejo de identificación para transporte e identificación. 
Tabla 8. Medición del cumplimiento de las buenas prácticas de almacenamiento en el área de Despacho.

\begin{tabular}{lccc}
\hline Area de Despacho & Cumple & No cumple & $\begin{array}{c}\text { Porcentaje de } \\
\text { Cumplimiento }\end{array}$ \\
\hline $\begin{array}{l}\text { Cuenta con un área separada, delimitada e } \\
\text { identificada }\end{array}$ & 1 & 0 & \\
$\begin{array}{l}\text { En el despacho de productos se verifica y se } \\
\text { registra }\end{array}$ & 1 & 0 & $100 \%$ \\
$\begin{array}{l}\text { Documentación que sustente el despacho } \\
\text { Que los productos a despachar corresponda a lo } \\
\text { solicitado }\end{array}$ & 1 & 0 & 0 \\
$\begin{array}{l}\text { Que el etiquetado del embalaje no se desprenda } \\
\text { tan facilmente }\end{array}$ & 1 & 0 & \\
$\begin{array}{l}\text { Que se identifiquen los lotes, series u otros } \\
\text { Se despachan los productos de acuerdo al }\end{array}$ & 1 & 0 & \\
sistema FIFO y/o FEFO & 1 & 0 & \\
TOTAL & 7 & 0 & \\
\hline
\end{tabular}

Por tanto, el porcentaje de cumplimiento de las Buenas prácticas de almacenamiento en el área de embalaje es del $0 \%$.

En la tabla 8 se puede apreciar en cuanto al nivel de cumplimiento en el área de despacho se tiene que se cumplieron en su totalidad los criterios (7), ya sea: Cuenta con un área separada, delimitada y identificada, en el despacho de productos se verifica y se registra documentación que sustente el despacho que los productos a despachar corresponda a lo solicitado, que el etiquetado del embalaje no se desprenda fácilmente, que se identifique los lotes, series u otros y que se despachen los productos de acuerdo al sistema FIFO y/o FEFO.

En consecuencia, existe un $100 \%$ de cumplimiento de Buenas Prácticas de Almacenamiento en el área de despacho.

\section{DISCUSIÓN}

Una vez interpretado mediante tablas y gráficos los resultados de esta investigación, realizado sobre las Buenas Prácticas de Almacenamiento en el área de almacén del Hospital Regional Docente Materno Infantil El Carmen y el Hospital Docente Clínico Quirúrgico Daniel Alcides Carrión, correspondiente a los hospitales nivel III especializada del MINSA; en esta investigación teninedo como objetivo evaluar el nivel y porcentaje de cumplimiento de las buenas prácticas de almacenamiento en hospitales nivel III especializada del MINSA- Huancayo, para cada uno de los criterios establecidos. Se considera un nivel alto de los valores mayores a $75 \%$ de cumplimiento, Nivel Medio a los valores entre $50 \%$ a $75 \%$ de cumplimiento y Nivel Bajo a los valores menores a 50\% de cumplimiento. encontrándose como resultado en este estudio que la buena práctica de almacenamiento alcanza un nivel medio de $65 \%$, lo que difiere con el trabajo de Cantos et al., quienes demostraron un nivel de cumplimiento alto del $74 \%$ (10).

De igual forma en esta investigación se consideró evaluar las buenas prácticas de almacenamiento en el área de almacén de los hospitales nivel III especializada del MINSA, encontrándose como resultado que se cumplieron 11 criterios como la existencia de la recepción, cuarentena, muestras de retención, aprobados/ almacenamiento, bajas/ rechazados, devoluciones, embalaje, despacho, productos controlados, área administrativa, servicios higiénicos, vestidores, material de limpieza y sin embargo existen puntos que se debe mejorar como las muestras de retención y embalaje, lo que se corrobora con el estudio de Villacra quien encontró como resultados que existen puntos que se deben mejorar (11), de igual manera se corrobora con el trabajo de Cortijo y Castillo, quienes donde según evaluación técnica diagnóstica determinó que existe cumplimiento parcial e incumplimiento en el control de los productos recibidos, registro de temperatura, distribución de los productos requeridos por los puntos de dispensación, conteo físico mensual, limpieza 
general una vez por mes, la capacitación y evaluación que se realizó al personal e internos de farmacia de forma continua y uso de implementos de seguridad para el manejo de los productos (12).

También, en este estudio fue importante evaluar las buenas prácticas de almacenamiento en el área de recepción de los hospitales nivel III especializada del MINSA, hallándose como resultado que cumplen un 100\% $(\mathrm{n}=5)$ de los indicadores establecido como: Área de recepción está separada, delimitada, identificada y equipada; cuenta con los procedimientos escritos para la recepción, cada producto ingresa con su respectiva documentación, se realiza la revisión del estado del embalaje, en la recepción, se verifica como mínimo, nombre, concentración, fecha de vencimiento y forma farmacéutica del producto farmacéutico, lo que se contrapone con el trabajo de Cantos et al., quienes demostraron que el nivel de cumplimiento para el factor áreas, obtuvo un nivel medio con el $74 \%$ (10); por tanto los resultados encontrados en nuestra investigación resulta favorable, cuando Polina, establece que esta área implica recibir el pedido, verificar su calidad, cantidad y empaque, donde los directores responsables son los encargados de hacer la recepción administrativa, comparando con la documentación que soporta el pedido y donde se hace la descripción del mismo como registro, código, precio, dosificación descripción, así como realizando la revisión cuidadosa para detectar cualquier anomalía que pueda alterar su contenido, de tal forma que si un medicamento no cumple con las especificaciones requeridas este no debe ser aceptado y posteriormente se debe hacer un informe él se deje en constancia del porqué de la inconformidad con el producto (13) .

En lo que respecta, para Evaluar el cumplimiento de las Buenas Prácticas de Almacenamiento en el área de cuarentena de los hospitales nivel III especializada del MINSA, se hallaron que se cumplieron 2 criterios como: Cuenta con un área separada, delimitada e identificada y si se realiza la verificación del registro de recepción certificado de análisis, obteniéndose un porcentaje de cumplimiento de Buenas Prácticas de Almacenamiento en el área de cuarentena del $50 \%$, lo que guarda cierta similitud con el estudio de Tenelema, en el trabajo de investigación donde se evaluó la incidencia de las Buenas Prácticas de Almacenamiento de medicamentos, hallándose en la evaluación final después de la aplicación de las BPA se obtuvo un $85 \%$ de cumplimiento de correcto almacenamiento (14).
Además, en este trabajo, fue necesario evaluar las Buenas Prácticas de Almacenamiento en el área de muestras de retención o contra muestras de los hospitales nivel III especializada del MINSA, hallándose que no cumplen el con el único indicador, que representa contar con un área separada, delimitada, identificada y registrada, por tanto el porcentaje de cumplimiento es del $0 \%$; lo que se corrobora con el estudio de Esparsa, quien encontró con respecto a la conservación de contramuestra, el cumplimiento se da de manera parcial, es decir que no se tomaban consideraciones necesarias para una eventual pesquisa o retiro del producto, por la cantidades recepcionadas para un análisis futuro (15).

En este estudio, además fue importante evaluar las Buenas Prácticas de Almacenamiento en el área de baja o rechazados, encontrándose un $100 \%(n=3)$ del porcentaje de cumplimiento de los indicadores establecidos como: Cuenta con un área separada, delimitada, identificada y restringida, cuenta con procedimientos operativos escritos por el proceso de baja de productos incluyendo la destrucción y se comunica a la autoridad sanitaria de su jurisdicción la destrucción de productos, lo que se contrapone con la investigación de Tenelema quien encontró como resultado que al identificar los parámetros de almacenamiento inicial de BPA que se utilizaba en la Farmacia siendo estos la infraestructura, organización, POE, devoluciones, área de baja y eliminación de medicamentos, ponen en riesgo la calidad y seguridad de los medicamentos (14), de igual forma con el trabajo de Esparsa, quien en su investigación, encontró que existe un cumplimiento parcial para el área de baja y destrucción de productos (15).

Finalmente al Evaluar las Buenas Prácticas de Almacenamiento en el área de embalaje y área de despacho de los hospitales nivel III especializada del MINSA en esta investigación se demostró que el porcentaje de cumplimiento de las Buenas prácticas de almacenamiento en el área de embalaje es del $0 \%$ y en el caso del área de despacho existe un $100 \%$, para los criterios de esta área ya sea: Cuenta con un área separada, delimitada e identificada, en el despacho de productos se verifica y se registra documentación que sustente el despacho que los productos a despachar corresponda a lo solicitado, que el etiquetado del embalaje no se desprenda fácilmente, que se identifique los lotes, series u otros y que se despachen los productos de acuerdo al sistema FIFO y/o FEFO, lo que se contrapone con el estudio de Esparsa, en el 
que encontró que existe un cumplimiento parcial para el área de embalaje y despacho (15), sin embargo se corrobora con el trabajo de, Cantos quien demostró que existe un porcentaje alto $100 \%$ en el factor técnicas de manejo y distribución de medicamentos, que incluye el área de despacho (10).

En consecuencia como se acredita en los formatos de autoinspección, los hospitales del MINSA de Huancayo con nivel medio de cumplimiento de Buenas Prácticas de Almacenamiento , no se encuentran adecuadas a la normatividad poniendo en riesgo la salud de la sociedad.

\section{CONCLUSIONES}

Los hospitales del MINSA de Huancayo, tienen un promedio de cumplimiento de las buenas prácticas de almacenamiento de nivel medio $(65 \%)$, encontrándose en nivel alto (entre 77 a 100\%) para las áreas de almacén, área de recepción, área de aprobados, área de baja y rechazados, área de devoluciones y el área de despacho; también de nivel medio $(50 \%)$ para el área de cuarentena; no obstante no cumplen para las áreas de muestras, contramuestras y el área de embalaje, lo que constituye una infracción de acuerdo a la normatividad vigente.

Las Buenas Prácticas de Almacenamiento en el área de almacén cumplieron 12 criterios y no cumpliéndose 1 criterio, siendo el porcentaje de cumplimiento del $77 \%$.

Las Buenas Prácticas de Almacenamiento en el área de recepción cumplieron 5 criterios, siendo el porcentaje de cumplimiento del 100\%.

Las Buenas Prácticas de Almacenamiento en el área de cuarentena se tiene que cumplieron 2 criterios de los 4 que existen, siendo el porcentaje de cumplimiento del $50 \%$.

Las Buenas Prácticas de Almacenamiento en el área de muestras de retención o contra muestras, no cumplen el único indicador : Cuenta con un área separada, delimitada, identificada y registrada; siendo el Porcentaje de cumplimiento del $0 \%$.

Las Buenas Prácticas de Almacenamiento en el área de aprobados se cumplieron 9 criterios de un total de 11 , siendo el porcentaje de cumplimiento del $82 \%$.
Las buenas prácticas de almacenamiento del área de baja o rechazados se tiene que cumplen los 3 indicadores establecidos, siendo el porcentaje de cumplimiento del $100 \%$.

Las buenas prácticas de almacenamiento en el área de devoluciones, se cumplieron 4 criterios de los 5 que existen, siendo el nivel de cumplimiento del $80 \%$.

Las Buenas Prácticas de Almacenamiento en el área de embalaje, se puede apreciar que no cumplieron los 6 criterios, que se requiere para esta área y siendo el porcentaje de cumplimiento de $0 \%$.

Las Buenas Prácticas de Almacenamiento en el área de despacho, se puede apreciar que se cumplieron los 7 criterios que se requiere para esta área y siendo el porcentaje de cumplimiento del $100 \%$.

\section{Correspondencia}

Diana Esmeralda Andamayo Flores Av. Giráldez 542, Huancayo 12001. Junin, Perú

\section{REFERENCIAS BIBLIOGRÁFICAS}

1. Chong M. Bases para la implementación de las Buenas Prácticas de Almacenamiento (BPA) en la farmacia universitaria de la Facultad de Farmacia y Bioquímica. Tesis. Perú: Universidad Mayor de San Marcos;2007.

2. Leyes D, López V. Diagnóstico sobre el nivel de cumplimiento de las Buenas Prácticas de Elaboración por parte de los procesos del servicio farmacéutico de la fundación valle del LILI.Tesis. Santiago De Cali: Universidad ICESI;2010.

3. Medrano J. Legislación Farmacéutica. Perú; 2011

4. Ministerio de Salud. Perú: Ley N ${ }^{\circ} 26842$ Ley General de Salud, Decreto Supremo $\mathrm{N}^{\circ} 010-97-\mathrm{SA}$. Reglamento para el Registro, control y Vigilancia Sanitaria de Productos Farmacéuticos y Afines Publicado; Lima: Ministerio de Salud; 2009;

5. Organización Mundial de la Salud. Cómo desarrollar y aplicar una política farmacéutica nacional [Internet].Ginebra: Organización Mundial de la Salud; 2003.

6. Congreso de la República. Perú: Ley de Productos Farmacéuticos, Dispositivos Médicos y Productos Sanitarios. Ley №29459. Normas legales; 2009. Lima. Ministerio de Salud; 2019.

7. Miranda L. Buenas Prácticas de Almacenamiento y Distribución. Guía de seguimiento. Perú; 2015.

8. Tobar F. Referenciación internacional sobre Políticas de Regulación de Medicamentos Llanos . Perú: 
Ministerio de salud; 2011.

9. Polit F, Hungler P. Investigación Científica en Ciencias de la Salud. Ciudad de México: Interamericana S.A.de C.V;1985.

10. Villacra V. Propuesta de implementación de buenas prácticas de almacenamiento en la bodega de medicamentos del hospital del IESS de Latacunga. Quito; 2013.

11. Cantos J, Cerna K. Nivel de Cumplimiento de las Buenas Prácticas de Almacenamiento en las Droguerías Inspeccionadas Por la Unidad Funcional de Regulación de Medicamentos Insumos y Drogas, Trujillo- 2013.Tesis. Perú: Universidad Nacional de Trujillo;2013.

12. Cortijo G, Castillo E. Implementación de las Buenas Prácticas de Almacenamiento en el almacén especializado de medicamentos del Hospital Belén de Trujillo, 2011. Scientia. 2012; 4(1):56-63.
13. Polina J. Recepción de Medicamentos 2013. Carvanme; 2013.

14. Tenelema J. Aplicación de Buenas Prácticas de Almacenamiento y su Incidencia en la Calidad de los Medicamentos e Insumos en la Farmacia del Sindicato de Choferes Profesionales, de la Provincia de Chimborazo.Tesis. Ecuador: Universidad Regional Autónoma de Los Andes; 2014.

15. Esparsa J. Propuesta de la implementación de Buenas Prácticas de Almacenamiento de una Droguería.Surco, Lima.2017.Tesis. Perú: Universidad Nacional de Trujillo; 2017.

Recibido: 14/12/2019 Aceptado: 15/07/2020 